\title{
Policy Statement on Antimicrobial Stewardship by the Society for Healthcare Epidemiology of America (SHEA), the Infectious Diseases Society of America (IDSA), and the Pediatric Infectious Diseases Society (PIDS)
}

\author{
Society for Healthcare Epidemiology of America; Infectious Diseases Society of America; \\ Pediatric Infectious Diseases Society
}

\begin{abstract}
Antimicrobial resistance has emerged as a significant healthcare quality and patient safety issue in the twenty-first century that, combined with a rapidly dwindling antimicrobial armamentarium, has resulted in a critical threat to the public health of the United States. Antimicrobial stewardship programs optimize antimicrobial use to achieve the best clinical outcomes while minimizing adverse events and limiting selective pressures that drive the emergence of resistance and may also reduce excessive costs attributable to suboptimal antimicrobial use. Therefore, antimicrobial stewardship must be a fiduciary responsibility for all healthcare institutions across the continuum of care. This position statement of the Society for Healthcare Epidemiology of America, the Infectious Diseases Society of America, and the Pediatric Infectious Diseases Society of America outlines recommendations for the mandatory implementation of antimicrobial stewardship throughout health care, suggests process and outcome measures to monitor these interventions, and addresses deficiencies in education and research in this field as well as the lack of accurate data on antimicrobial use in the United States.
\end{abstract}

Infect Control Hosp Epidemiol 2012;33(4):322-327

It is widely acknowledged that the availability of effective antimicrobial therapy is one of the most important developments in clinical medicine. The harnessing of antibacterial agents for clinical use began during the 1930s-1940s, when sulfonamides, penicillin, and streptomycin became available. It was recognized early that bacteria exposed to antimicrobial agents evolved strategies to survive them, raising the concern that these agents should be used carefully in order to preserve their effectiveness. Sir Alexander Fleming made the following cautionary statements on June 26,1945, in a New York Times article "... the microbes are educated to resist penicillin and a host of penicillin-fast organisms is bred out....In such cases the thoughtless person playing with penicillin is morally responsible for the death of the man who finally succumbs to infection with the penicillin-resistant organism. I hope this evil can be averted."

In the latter half of the twentieth century, a large number of antimicrobial products, including synthetic compounds, became available for clinical use. The ability to control infections through the use of antimicrobial agents has had a major impact in all clinical areas, but particularly in surgery, transplantation medicine, oncology, and intensive care medicine. Penicillin resistance in Staphylococcus aureus was initially detected in clinical specimens in 1945, and resistance to methicillin emerged in $1961 .{ }^{2,3}$ By 1999 , methicillin resistance in $S$. aureus was observed in over $53 \%$ of $S$. aureus isolates obtained from patients in intensive care units in a US surveillance system. ${ }^{4}$ Strains of methicillin-resistant $S$. aureus (MRSA) emerged in the 1990s as causes of infections in community-residing patients and became common in most geographic areas in the United States in 2000.5-7

The past 30 years have brought multidrug-resistant pneumococci, gonoccocci, and Salmonella spp. and extremely drug-resistant tuberculosis to patients in the community. ${ }^{8-11}$ Vancomycin-resistant enterococci and vancomycin-resistant $S$. aureus have also emerged. ${ }^{12-14}$ Extremely drug-resistant gram-negative bacteria, such as carbapenemase-producing Klebsiella pneumoniae and other carbapenem-resistant Enterobacteriaceae spp., extended-spectrum beta-lactamase-producing Enterobacteriaceae, Pseudomonas aeruginosa, and Acinetobacter baumanii have spread widely among patients in healthcare settings; in some cases these pathogens have been panresistant, that is, resistant to all available antibiotics. ${ }^{15-22}$

Unfortunately, during the last decade there has also been a dramatic drop in the development and approval of new antibacterial agents. ${ }^{23}$ The antimicrobial armamentarium has been depleted and our ability to treat infectious diseases has been severely compromised. Resistant infections not only re- 
sult in increased morbidity and mortality but also dramatically increase healthcare costs. ${ }^{24-28}$ It is ironic that in the twenty-first century we are encountering bacterial infections for which we have no treatment. A multifaceted approach is necessary to prevent, detect, and control the emergence of antimicrobial-resistant organisms. This includes ensuring the availability of adequate and appropriate therapeutic agents, the existence of diagnostic capacity to rapidly and reliably detect specific pathogens and their antimicrobial susceptibilities, and the promotion of robust infection prevention, control, and antimicrobial stewardship programs. This document focuses on issues relating to antimicrobial stewardship. Other issues important to the emergence, transmission, and management of antimicrobial resistance are addressed elsewhere. ${ }^{29,30,34}$

The Society for Healthcare Epidemiology of America (SHEA) and the Infectious Diseases Society of America (IDSA) recognized these needs in 1997 with the publication of "Guidelines for the Prevention of Antimicrobial Resistance in Hospitals." ${ }^{" 1,32}$ In 2007, these societies promoted the concept of antimicrobial stewardship when they issued "Guidelines for Developing an Institutional Program to Enhance Antimicrobial Stewardship," ${ }^{33}$ which discusses the development of multidisciplinary teams in acute care settings to review and improve antimicrobial use and improve patient care. A recent IDSA policy paper titled "Combating Antimicrobial Resistance: Policy Recommendations to Save Lives" has been issued. ${ }^{34}$ It urges a strengthening of US efforts to improve prevention and control efforts, including the adoption of antimicrobial stewardship programs in all US healthcare facilities. Other recommendations include research to define optimal elements and goals of antimicrobial stewardship programs in different healthcare settings, expanded educational efforts on antimicrobial stewardship, novel mechanisms to prevent the overprescription of newly approved antibacterial agents, and the development of new antibacterial therapies, vaccines, and rapid, point-of-care diagnostic tests that would enable appropriate care, including the avoidance of antibacterial agents for viral etiologies.

In recognizing the importance of antimicrobial stewardship as it relates to children, the Pediatric Infectious Diseases Society (PIDS) has developed an annual meeting to address the importance of antimicrobial stewardship for children. PIDS and SHEA have partnered to form a joint antimicrobial stewardship committee to address inpatient antibiotic use, outpatient antibiotic use, antimicrobial stewardship in special populations, education involving antibiotic use, and research on antibiotic use and stewardship. In this joint SHEA-IDSAPIDS position paper, we focus on the need for public policy around the issue of antimicrobial stewardship.

\section{DEFINITION}

Antimicrobial stewardship refers to coordinated interventions designed to improve and measure the appropriate use of an- timicrobial agents by promoting the selection of the optimal antimicrobial drug regimen including dosing, duration of therapy, and route of administration. The major objectives of antimicrobial stewardship are to achieve best clinical outcomes related to antimicrobial use while minimizing toxicity and other adverse events, thereby limiting the selective pressure on bacterial populations that drives the emergence of antimicrobial-resistant strains. Antimicrobial stewardship may also reduce excessive costs attributable to suboptimal antimicrobial use.

\section{RECOMMENDATIONS}

\section{Antimicrobial Stewardship Programs Should Be Required through Regulatory Mechanisms}

At present there are no national or coordinated legislative or regulatory mandates designed to optimize the use of antimicrobial therapy through antimicrobial stewardship. Legislation is also limited at the state level.

California Senate Bill 739 mandated that by January 1, 2008, the California Department of Public Health (CDPH) require that all general acute care hospitals develop a process for evaluating the judicious use of antibiotics, the results of which shall be monitored jointly by appropriate representatives and committees involved in quality improvement activities. While this is the first legislative mandate of its kind, it does not specify that hospitals must intervene to improve antimicrobial use, that is, to have an antimicrobial stewardship program. Thus, the $\mathrm{CDPH}$ is learning that given the nonspecific wording used in the mandate, many hospitals are able to meet this requirement without having an antimicrobial stewardship program that meets the objectives as defined above. On the other hand, successful antimicrobial stewardship programs in California are varied, utilizing different combinations of staff, strategies, and criteria; therefore, changing the regulation to be too specific may prevent resource-limited hospitals from developing robust antimicrobial stewardship programs on the basis of facility-specific attributes.

In a preliminary assessment of acute care hospitals in California, $23 \%$ of hospitals reported being influenced to start an antimicrobial stewardship program because of Senate Bill 739. Lessons learned from statutory requirements in California include that regulatory mandates are important in convincing hospital administration to fund and staff antimicrobial stewardship programs. It is important to use the wording "antimicrobial stewardship program" in the regulation, as defined above, but it is also important to allow hospitals the flexibility to define how their facility can best meet the objectives of an antimicrobial stewardship program. Inasmuch as current legislation is limited to a single state and focuses only on institutional evaluation of antimicrobial use in hospitals, we support broad implementation of comprehensive antimicrobial stewardship programs across all healthcare settings. Antimicrobial resistance is a critical issue that signifi- 
cantly impacts healthcare quality, patient safety, and public health. As such, antimicrobial stewardship and other efforts to limit the emergence and transmission of antimicrobial resistance must be viewed as the fiduciary responsibility of all healthcare institutions across the continuum of care.

SHEA, IDSA, and PIDS recommend that the Centers for Medicare and Medicaid Services (CMS) require participating healthcare institutions to develop and implement antimicrobial stewardship programs. This can be achieved by incorporating the requirement into existing regulations via expansion of interpretive guidelines of the relevant regulation(s). All healthcare facilities, including hospitals, long-term care facilities, long-term acute care facilities, ambulatory surgical centers, and dialysis centers should develop and implement an antimicrobial stewardship plan that is modeled after the IDSA and SHEA "Guidelines for Developing an Institutional Program to Enhance Antimicrobial Stewardship." ${ }^{33}$ Minimum requirements for the program should include:

A. Creation of a multidisciplinary interprofessional antimicrobial stewardship team that is physician directed or supervised. At a minimum, 1 or more members of the team should have training in antimicrobial stewardship. The number of team members may vary on the basis of the size and complexity of the facility. Team members should include but are not limited to:

- A physician.

- A pharmacist.

- A clinical microbiologist.

- An infection preventionist.

B. A formulary limited to nonduplicative antibiotics with demonstrated clinical need.

C. Institutional guidelines for the management of common infection syndromes.

D. Additional interventions to improve the use of antimicrobials, including those designed to detect and eliminate:

- Multidrug regimens with unnecessarily redundant antimicrobial spectra.

- Antibiotic therapy for the management of nonbacterial syndromes or cultures that represent contamination or routine colonization.

- Empiric regimens that are either inadequately or excessively broad spectrum for infection syndromes.

- Regimens that do not adequately treat infections caused by culture-confirmed pathogens.

E. Processes to measure and monitor antimicrobial use at the institutional level for internal benchmarking.

F. Periodic distribution of a facility-specific antibiogram indicating the rates of relevant antibiotic susceptibilities to key pathogens.

CMS should seek to improve the development, imple- mentation, and monitoring of antimicrobial stewardship plans and programs over time by requiring additional activities. Such measures may include:

A. Reporting to the Antimicrobial Use and Resistance option of the Medication-Associated Module of the Centers for Disease Control and Prevention's (CDC's) National Healthcare Safety Network (NHSN).

B. Prospective surveillance and concurrent intervention for the inappropriate use of antimicrobial agents.

C. National benchmarking of antimicrobial use at the institutional level based on acuity of care and patient mix.

D. Relevant future outcome measures, which may include:

- Prevalence and incidence of drug-resistant phenotypes among common clinical pathogens (eg, carbapenemresistant Enterobacteriaciae, carbapenem-resistant Acinetobacter, extensively drug-resistant Pseudomonas, MRSA).

- Incidence of diarrhea caused by Clostridium difficile.

- Rates of adverse antimicrobial drug reactions and interactions.

\section{Antimicrobial Stewardship Should Be Monitored in Ambulatory Healthcare Settings}

Effective mechanisms do not currently exist to optimize antimicrobial use in ambulatory healthcare settings. Ambulatory settings include but are not limited to outpatient clinical practices, ambulatory surgical centers, and dialysis centers. Inasmuch as these settings account for a significant portion of the antimicrobial use in the United States and there is ample evidence that antimicrobial resistance is emerging as a problem in the community, effective and efficient antimicrobial stewardship initiatives must be developed for these settings. Additionally, such a focus coincides with and complements the implementation of tier 2 of the Department of Health and Human Services' Action Plan to Prevent HealthcareAssociated Infections. ${ }^{35}$ Therefore, SHEA, IDSA, and PIDS believe that federal agencies such as the Agency for Healthcare Research and Quality (AHRQ), the Office of the National Coordinator for Health Information Technology, CMS, the National Institutes of Health (NIH), and CDC should fund pilot projects designed to develop and implement antimicrobial stewardship in ambulatory settings. We believe that expanded utilization of electronic health records (EHRs) offers great potential in this regard. Areas of study may include:

- Integration of clinical decision support technology into EHRs.

- Integration of clinical decision support technology into eprescribing mechanisms.

If these interventions are validated in these pilot project 
programs, then we support the subsequent integration in the CMS requirement for meaningful use of EHRs.

\section{Education about Antimicrobial Resistance and Antimicrobial Stewardship Must Be Accomplished}

SHEA, IDSA, and PIDS believe that significant knowledge deficits in the areas of antimicrobial resistance and antimicrobial stewardship are prevalent among healthcare providers in the United States. Educational programs should be developed for those in training programs as well as for all prescribing clinicians that teach about the science behind, the principles of, and the tools essential for the practice of effective antimicrobial stewardship. Education about antimicrobial resistance and stewardship should be incorporated into curriculum requirements for medical students and postgraduate residents and fellows. It is crucial that currently practicing clinicians become proficient in these areas. In addition to ensuring that these areas are included in curricula and programs for those in training, there are a number of ways in which proficiency may be accomplished for practicing clinicians, including partnering with specialty societies and the Food and Drug Administration (FDA) to provide educational resources. Moreover, as a part of the drug-review process, pharmaceutical sponsors should include a plan to educate healthcare providers about both the optimal use of the drug and precautions that reduce the emergence of antimicrobial resistance.

Individual facilities should be responsible for supporting the education of the members of the antimicrobial stewardship team. Antimicrobial stewardship is a patient safety issue and a public health issue and must be taken seriously in all aspects of the continuum of patient care. Additionally, because of the gravity of the problems with antimicrobial resistance that confront society and the paucity of readily available clinical solutions, SHEA, IDSA, and PIDS support appropriations to fund these education initiatives.

\section{Antimicrobial Use Data Should Be Collected and Readily Available for Both Inpatient and Outpatient Settings}

Accurate and readily available data to track and benchmark antimicrobial use is currently lacking in the United States. The United States is unique among developed countries in that there is no access to these data. We believe that these data are critical to being able to monitor antimicrobial use and its relationship to antimicrobial resistance, and therefore we advocate for a reliable and accurate national system for collecting data on antimicrobial use. When this system is developed, validated, and operationalized, antimicrobial use can be benchmarked, and these data should be utilized as a component of an incentive-based payment system. Reporting to the Antimicrobial Use and Resistance option of the Med-
ication-Associated Module of the CDC's NHSN may accomplish this goal.

\section{Research on Antimicrobial Stewardship Is Needed}

Significant knowledge gaps exist in our understanding of antimicrobial resistance and interventions to limit both the emergence and the transmission of resistance, as well as in our ability to measure associated impacts and clinical outcomes in these areas. SHEA, IDSA, and PIDS believe that we must refocus translational research efforts in order to answer these questions that are critical to our future ability to effectively treat and manage infectious diseases in the United States. All areas of the translational research paradigm must be addressed, ranging from basic bench science and epidemiologic investigations (T0) to implementation science (T4). Two primary issues of equal importance must be considered in this regard: (1) the benchmarking of antimicrobial use within and between institutions, and the most effective and efficient interventions to optimize these measures; and (2) the development of clear, well-defined, and validated process and outcome measures that may be utilized to assess the clinical impact of stewardship efforts. Initial research proposals should focus on but not necessarily be limited to the following critical issues:

A. Research is needed to develop a standardized definition of both appropriate and inappropriate antimicrobial use, clear and unambiguous measures of such use, and the risk factors that promote the unnecessary overuse and abuse of antimicrobial therapy. Standardized data collection tools should also be developed to facilitate measurement and interpretation of antimicrobial use data by both government and professional agencies. Furthermore, delineation of the primary drivers of inappropriate antimicrobial use and the relative contribution of individual risk factors that contribute to this outcome are essential to the development of the most effective interventions to prevent these prescriptions.

B. Patient-centered outcomes research is needed to determine the most effective and cost-efficient deployment of antimicrobial stewardship interventions in different healthcare settings. To date, research in these areas has been plagued by poor study design issues and an absence of standardized definitions. Specifically, current research efforts demonstrate selection biases, insufficient power to answer proposed questions, varying duration of interventions, failure to deal with confounding variables, failure to measure compliance with the intervention processes, and a lack of generalizability. Therefore, SHEA, IDSA, and PIDS recommend using robust study designs that include multicenter randomized-cluster-designed studies that compare stewardship interventions across various healthcare settings as well as the impact of these interventions 
on epidemic and endemic antimicrobial resistance within single and across multiple institutions.

C. Research is needed to develop and validate clear and welldefined process and outcome measures that may be utilized to assess the impact of antimicrobial stewardship interventions both within and across various healthcare settings. While it is critical to understand the impact of antimicrobial stewardship on epidemic and endemic resistance rates both within and between healthcare institutions, we must also develop and validate additional surrogate markers of success. Such measures may include but are not limited to rates of $C$. difficile infection, time to administration of appropriate therapy, adverse drug reactions or interactions related to antimicrobial therapy, drugs administered to patients with documented allergies, multidrug regimens with redundant antimicrobial spectra, regimens that are either inadequate or excessive, and duration of intensive care and overall hospitalization for patients treated with antimicrobials.

D. SHEA, IDSA, and PIDS believe that it is critical that the United States develop accurate measures of antimicrobial use such as those available in most other developed countries. Such measures can be used to track antimicrobial utilization and correlate such use with emerging antimicrobial resistance patterns. Therefore, an accurate understanding of antimicrobial use data may be used to develop and implement regional targeted interventions to limit the transmission of emerging multidrug-resistant organisms. As noted above, these data may be obtained through annual national point-prevalence surveys of antimicrobial use and/or by reporting to the Antimicrobial Use and Resistance option of the Medication-Associated Module of CDC's NHSN. However, research is needed to determine the validity of both data sets across the continuum of care. For instance, one may prove to be a more accurate representation of antimicrobial use in hospitalized patients whereas the other may more precisely reflect antimicrobial use in the community.

E. Research is required to understand the impact of the use of generic versus branded antimicrobial agents on how antibiotics are used.

F. Research is needed to develop and evaluate accurate, easyto-use, rapid point-of-care diagnostic tests so that antibacterial therapy can be avoided when a viral etiology is identified and used appropriately as indicated by specific bacterial etiologies. The scientific issues surrounding the development and use of such rapid diagnostics are discussed in 2 other IDSA position papers. ${ }^{34,36}$ In addition, further research into the use of biomarkers (such as procalcitonin) that can help to distinguish bacterial from viral disease would be useful in optimizing the use of antibacterial agents, including determining the appropriate duration of therapy. ${ }^{37}$

Finally, it is imperative that the appropriate federal agen- cies, such as CDC, AHRQ, FDA, and NIH, receive adequate appropriations to fund these research efforts.

\section{ACKNOWLEDGMENTS}

The boards of directors of SHEA, IDSA, and PIDS wish to recognize the many experts who contributed to the development of this joint statement, including, for SHEA: Drs. Neil Fishman, Jan Patterson, Lisa Saiman, Arjun Srinivasan, Kavita K. Trivedi, Trevor van Schooneveld; for IDSA: Drs. Ruth Lynfield, Dale Gerding, Edward Septimus, David Schwartz, and Robert Daum; and for PIDS: Drs. Janet A. Englund, Christopher J. Harrison, John S. Bradley, and Jason Newland. In particular, the SHEA, IDSA, and PIDS boards would like to extend their deep appreciation to Drs. Fishman, Lynfield, and Harrison for shepherding the development of the joint policy statement.

Potential conflicts of interest: Societal policies regarding disclosure of potential conflicts of interest can be obtained by contacting each society listed as an author.

Address correspondence to Neil Fishman, MD, University of Pennsylvania Health System, 3400 Spruce Street, Penn Tower, Suite 101, Philadelphia, PA 19104 (neil.fishman@uphs.upenn.edu).

\section{R E F E R E N C ES}

1. Penicillin finder assays its future. New York Times. 26 June 1945: 21.

2. Spink WW, Ferris V. Quantitative action of penicillin inhibitor from penicillin-resistant strain of Staphylococcus. Science 1945; 102:221-223.

3. Barrett FF, McGehee RF, Finland M. Methicillin-resistant Staphylococcus aureus at Boston City Hospital. N Engl J Med 1968; 279:441-448.

4. Centers for Disease Control and Prevention NNIS System. National Nosocomial Infections Surveillance (NNIS) system report, data summary from January 1992-April 2000; issued June 2000. Am J Infect Control 2000;28:429-448.

5. Herold BC, Immergluck LC, Maranan MC, et al. Communityacquired methicillin-resistant Staphylococcus aureus in children with no identified predisposing risk. JAMA 1998;279:593-598.

6. Naimi TS, LeDell KH, Como-Sabetti K, et al. Comparison of community- and health care-associated methicillin-resistant Staphylococcus aureus infection. JAMA 2003;290:2976-2984.

7. Deleo FR, Otto M, Kreisworth BN, Chambers HF. Communityassociated meticillin-resistant Staphylococcus aureus. Lancet 2010;375:1557-1568.

8. Lynch JP 3rd, Zhanel GG. Streptococcus pneumoniae: epidemiology and risk factors, evolution of antimicrobial resistance, and impact of vaccines. Curr Opin Pulm Med 2010;16:217-225.

9. Deguchi T, Nakane K, Yasuda M, Maeda S. Emergence and spread of drug resistant Neisseria gonorrhoeae. J Urol 2010;184: 851-858.

10. Parry CM, Threlfall EJ. Antimicrobial resistance in typhoidal and nontyphoidal salmonellae. Curr Opin Infect Dis 2008;21: 531-538.

11. Jacobson KR, Tierney DB, Jeon CY, Mitnick CD, Murray MB. Treatment outcomes among patients with extensively drugresistant tuberculosis: systematic review and meta-analysis. Clin Infect Dis 2010;51:6-14.

12. Bonten MJ, Willems R, Weinstein RA. Vancomycin-resistant en- 
terococci: why are they here, and where do they come from? Lancet Infect Dis 2001;1:314-325.

13. Chavers LS, Moser SA, Benjamin WH, et al. Vancomycinresistant enterococci: 15 years and counting. J Hosp Infect 2003; 53:159-171.

14. Hiramatsu K. Vancomycin-resistant Staphylococcus aureus: a new model of antibiotic resistance. Lancet Infect Dis 2001;1:147-155.

15. Nordmann P, Cuzon G, Naas T. The real threat of Klebsiella pneumoniae carbapenemase-producing bacteria. Lancet Infect Dis 2009;9:228-236.

16. Ho J, Tambyah PA, Paterson DL. Multiresistant gram-negative infections: a global perspective. Curr Opin Infect Dis 2010;23: 546-553.

17. Oteo J, Pérez-Vázquez M, Campos J. Extended-spectrum betalactamase producing Escherichia coli: changing epidemiology and clinical impact. Curr Opin Infect Dis 2010;23:320-326.

18. Garnacho-Montero J, Amaya-Villar R. Multiresistant Acinetobacter baumannii infections: epidemiology and management. Curr Opin Infect Dis 2010;23:332-339.

19. Zavascki AP, Carvalhaes CG, Picão RC, Gales AC. Multidrugresistant Pseudomonas aeruginosa and Acinetobacter baumannii: resistance mechanisms and implications for therapy. Expert Rev Anti Infect Ther 2010;8:71-93.

20. Falagas ME, Rafailidis PI, Matthaiou DK, Virtzili S, Nikita D, Michalopoulos A. Pandrug-resistant Klebsiella pneumoniae, Pseudomonas aeruginosa and Acinetobacter baumannii infections: characteristics and outcome in a series of 28 patients. Int $J$ Antimicrob Agents 2008;32:450-454.

21. Chen LF, Chopra T, Kaye KS. Pathogens resistant to antibacterial agents. Infect Dis Clin N Am 2009;23:817-845.

22. Rice LB. The clinical consequences of antimicrobial resistance. Curr Opin Microbiol 2009;12:476-481.

23. Boucher HW, Talbot GH, Bradley JS, et al. Bad bugs, no drugs: no ESKAPE! an update from the Infectious Diseases Society of America. Clin Infect Dis 2009;48:1-12.

24. Lautenbach E, Weiner MG, Nachamkin I, Bilker WB, Sheridan A, Fishman NO. Imipenem resistance among Pseudomonas aeruginosa isolates: risk factors for infection and impact of resistance on clinical and economic outcomes. Infect Control Hosp Epidemiol 2006;27:893-900.

25. Roberts RR, Hota B, Ahmad I, et al. Hospital and societal costs of antimicrobial-resistant infections in a Chicago teaching hospital: implications for antibiotic stewardship. Clin Infect Dis 2009;49:1175-1184.

26. Patel G, Huprikar S, Factor SH, Jenkins SG, Calfee DP. Outcomes of carbapenem-resistant Klebsiella pneumoniae infection and the impact of antimicrobial and adjunctive therapies. Infect Control Hosp Epidemiol 2008;29:1099-1106.

27. Filice GA, Nyman JA, Lexau C, et al. Excess costs and utilization associated with methicillin resistance for patients with Staphylococcus aureus infection. Infect Control Hosp Epidemiol 2010;31: 365-373.

28. Mauldin PD, Salgado CD, Hansen IS, Durup DT, Bosso JA. Attributable hospital cost and length of stay associated with health care-associated infections caused by antibiotic-resistant gram-negative bacteria. Antimicrob Agents Chemother 2010;54: 109-115.

29. Infectious Diseases Society of America. The $10 \times 20$ Initiative: pursuing a global commitment to develop 10 new antibacterial drugs by 2020. Clin Infect Dis 2010;50:1081-1083.

30. Infectious Diseases Society of America. Antimicrobial resistance. http://www.idsociety.org/AR_Policy/. Accessed October 27, 2010.

31. Shlaes DM, Gerding DN, John JF Jr, et al. Society for Healthcare Epidemiology of America and Infectious Diseases Society of America Joint Committee on the Prevention of Antimicrobial Resistance: guidelines for the prevention of antimicrobial resistance in hospitals. Infect Control Hosp Epidemiol 1997;18: 275-291.

32. Shlaes DM, Gerding DN, John JF Jr, et al. Society for Healthcare Epidemiology of America and Infectious Diseases Society of America Joint Committee on the Prevention of Antimicrobial Resistance: guidelines for the prevention of antimicrobial resistance in hospitals. Clin Infect Dis 1997;25:584-599.

33. Dellit TH, Owens RC, McGowan JE Jr, et al. Infectious Diseases Society of America and the Society for Healthcare Epidemiology of America guidelines for developing an institutional program to enhance antimicrobial stewardship. Clin Infect Dis 2007;44: 159-177.

34. Spellberg B, Blaser M, Guidos RJ, et al for the Infectious Diseases Society of America. Combating antimicrobial resistance: policy recommendations to save lives. Clin Infect Dis 2011;52(suppl 5): S397-S428.

35. Department of Health and Human Services. HHS action plan to prevent healthcare-associated infections. http://www.hhs.gov/ ash/initiatives/hai/actionplan/index.html. Accessed October 27, 2010.

36. Gilbert DN, Spellberg B, Bartlett JG for the Infectious Diseases Society of America. Position paper: an unmet medical need: rapid molecular diagnostic tests for respiratory tract infections. Clin Infect Dis 2011;52(suppl 4):S384-S395.

37. Gilbert DN. Use of plasma procalcitonin levels as an adjunct to clinical microbiology. I Clin Microbiol 2010;48:2325-2329. 